\section{Current management of intra- mural duodenal hematoma in alcohol induced pancreatitis}

\author{
Florian Bert, Thomas Jun, \\ Siegbert Rossol \\ Department of Internal Medicine, \\ Krankenhaus Nordwest, Frankfurt am \\ Main, Germany
}

\begin{abstract}
Intramural duodenal hematomas (IDH) have been rarely associated with pancreatic diseases. Conservative treatment is recommended, but course of disease can be life threatening, serious complications may occur (i.e. duodenal perforation) with imperative surgery. The management of diagnostic and treatment in IDH has improved over the years. Computed tomography (CT) and endoscopic ultrasound are excellent tools for diagnosis and follow up of IDH. We report a case of a 31-year-old alcoholic who presented with vomiting, exsiccosis, hypochondriac pain and positive shock index. Esophagogastroduodenoscopy showed gastric outlet obstruction caused by obliterating tumor of bulbus duodeni. Initial suspicion was malign tumor of the duodenum confirmed by native CT and histology. Further diagnostic using EUS-guided aspirate resulted in IDH. By conservative management with nasogastric decompression and digestive rest the patient recovered. In course of disease the hematoma got smaller, but parts were still seen in CT 6 month later.
\end{abstract}

\section{Introduction}

Intramural duodenal hematoma (IDH) is a rare pathology that involves a generally self-limited hemorrhage located on the submucosal layer of the wall. ${ }^{1-3}$ MacLauchlan described a fist case of IDH at autopsy in the Lancet 1838, later on Henoch-Schönlein and Von Khautz in context with blood dyscrasia. ${ }^{4,5}$ In $70-75 \%$ of the cases blunt abdominal trauma is the main cause of IDH. Other cases include endoscopic procedures or peptic ulcera. ${ }^{6}$ Spontaneous IDH is more related to the use of anticoagulant therapy or coagulation disorders (i.e. Von Willebrand disease, hemophilia). ${ }^{3}$ Disease course can be life threatening by complications, including upper gastrointestinal tract obstruction, jejunal intussusception, duodenal perforation or septic shock. A small percentage is described in context with pancre- atic processes like ectopic tissue of pancreas, pancreatitis and especially malignant tumors. ${ }^{7,8}$ The exact mechanism of pancreatic-induced IDH is still unclear. When complications appear surgical interventions are often necessary.

Diagnosis of IDH relies on clinical suspicion and accurate radiological studies like contrast-enhanced computed tomography (CT). ${ }^{9}$ But in spite of diagnostic possibilities early diagnosis of IDH can be difficult.

Over the year's paradigm towards management in IDH and its therapy - surgical intervention versus conservative therapy changed.

We report a case of intramural duodenal hematoma that happened in context with alcohol-induced pancreatitis and give a review about the current trend for this rare condition.

\section{Case Report}

A 31-year-old male presented with exsiccosis, hematemesis and belt-like abdominal pain in the interdisciplinary emergency unit (ED) of our hospital. He reported 3-5 daily episodes of watery, non-bloody diarrhea over a period of 5 days and daily alcohol intake of 1 bottle Vodka until 5 days before presentation. He lost about $5 \mathrm{~kg}$ weight in 2 weeks. The patient failed conservative management including medication intake like antibiotic therapy and denied recent travels or sick contact, prior exposure to health care environments. On presentation, the patient showed a positive shock index ( $>1 / \mathrm{mmHg}$ min) with orthostatic hypotension (systolic pressure: 90 $\mathrm{mmHg}$ and diastolic: $55 \mathrm{mmHg}$ ) and heart frequency up to 110 beats per minute. Physical examination was unremarkable except for the finding of dehydration and tenderness in epigastric region. Laboratory data were pertinent for: leukocytes: 16.96/nL, hemoglobin: $11.5 \mathrm{~g} / \mathrm{dL}$; mean corpuscular volume (MCV): $104.3 \mathrm{fl}$, calcium: $2.11 \mathrm{mmol} / \mathrm{L}$, uric acid: $10.8 \mathrm{mg} / \mathrm{dL}$, creatinine: $2.0 \mathrm{mg} / \mathrm{dL}$, glomerular filtration rate (GFR): $<50 \mathrm{~mL} / \mathrm{min}$; glucose: 233 $\mathrm{mg} / \mathrm{dL}$, albumin: $3442 \mathrm{mg} / \mathrm{dL}$, c-reactive protein: $28 \mathrm{mg} / \mathrm{L}$, aspartate aminotransferase: $95 \mathrm{U} / \mathrm{L}$, alanine aminotransferase: $57 \mathrm{U} / \mathrm{L}$, gamma-glutamyltransferase: 1160 $\mathrm{U} / \mathrm{L}$, gamma-glutamyltransferase: $154 \mathrm{U} / \mathrm{L}$, lipase: $1189 \mathrm{U} / \mathrm{L}$, alcohol: <0.1 o/oo. Other initial workup including serum thyroid stimulating hormone, hepatitis A, B, C and E screening, electrophoresis, quantitative immunoglobulin's, stool cultures, stool Clostridium difficile toxin assay, stool ova and parasites was unrevealing as well as the
Correspondence: Florian Bert, Department of Internal Medicine, Krankenhaus Nordwest Steinbacher Hohl 2-26, 60488 Frankfurt am Main, Germany,

Tel.: +49.69.7601.4147 - Fax: +49.69.7601.3614. E-mail: florianbert@freenet.de

Key words: pancreatitis, duodenal hematoma, complications, adenocarcinoma.

Acknowledgements: the authors thank Prof. Dr. H.M. Altmannsberger for allocating histological pictures.

Contributions: FB is the article guarantor and prepared the manuscript; TJ and SR edited and proved the manuscript

Conflicts of interest: the authors declare no potential conflict of interest.

Received for publication: 4 August 2017.

Revision received: 26 October 2017.

Accepted for publication: 26 October 2017.

This work is licensed under a Creative Commons Attribution NonCommercial 4.0 License (CC BY-NC 4.0).

x-ray of the chest. After recovery from acute exsiccosis by application of sodium chloride liquid $0.9 \%$ via central vein catheter we undertake additional esophagogastro-duodenoscopy (EGD). The tissue of the esophagus was macerated, coated with hematin and fibrin corresponding reflux esophagitis D (Los Angeles Classification). These lesions were caused by overfilled stomach with nearly $1100 \mathrm{~mL}$ liquid aspirated by endoscope. By pushing forward the tissue of bulbus duodeni appeared necrotic and duodenal lumen was completely obliterated by tumor dubious origin with rough, vulnerable surface. We took tissue samples, and to specify the lesion native computed tomography (CT) of abdomen and pelvis was performed. Contrast-enhanced CT was impossible because of reduced GFR and elevated creatinine. The scan showed an extensive, homogenous mass of the duodenal wall from duodenal part I up to II (Figure 1).

Initial differential diagnosis was lymphoma or gastrointestinal stromal tumor (GIST). Secondary finding was an exsudative pancreatitis of the pancreas head with little ascites matching to serological elevated lipase. Additional ultrasound of the abdomen with supplemental elastography (acoustic radiation force imaging) revealed fatty liver without sings of liver fibrosis or portal hypertension. As seen in the scan the duodenal wall was thickened up to $5 \mathrm{~cm}$ and pancreas head was swollen with little 
ascites. We monitored the patient at the intensive care station and supported him parenteral while fasting. He was treated with proton pump inhibitors and dimenhydrinate additionally.

The histological examination of duodenal biopsies gave hints for malignant cells corresponding adenocarcinoma, but without clear proof (Figure 2). Re-biopsy was recommended.

A subsequent EGD three days later validated the post-pyloric stenosis. This time the surface of the tissue was smooth with a reddish aspect. New biopsies were taken. This time the passage of stenosis with 8.8 $\mathrm{mm}$ endoscope was possible and we placed a three luminal, jejunal tube for enteral feeding and draining gastric liquid. To get more information about the duodenal wall tumor and its origin we used endoscopic ultrasound (EUS). Here the pancreas head was edematous with a small margin of ascites and the duodenal wall was now inhomogenic, without suspicious vascularization but parts of liquid. We took EUSguided fine needle aspiration of the wall and direct cytological evaluation of the aspirate showed coagulated hematoma. Thus IDH was the diagnosis (Figure 3). Further histological examination of the duodenal biopsies confirmed IDH and excluded malignancy.

In the course of disease the clinical status of the patient became better while intensive care management. We observed IDH and pancreatitis by ultrasound and serological markers. No complications like intraabdominal bleeding or perforation occurred. The duodenal hematoma became more liquid. EGD on day 10 showed an obvious regression of the hematoma. The parameters recovered after several days with no signs of further complications like cystic lesion of pancreas and patient demission was possible.

Contrast-enhanced follow-up CT five weeks later showed further regression of the hematoma, but it was still there. Half a year later the patient presented again in our ED with clinical and serological signs of acute pancreatitis caused by alcohol intake. The additional CT scan demonstrated a mild pancreas head pancreatitis without ascites. Residues of the former duodenal wall hematoma were still seen, without liquid but solid parts. The additional EGD was without pathologic results. Deep biopsies of the duodenal wall were unsuspicious for malignancy.

\section{Discussion}

We presented a case of intramural duo- denal hematoma in context with alcohol induced acute pancreatitis. The young patient was alcoholic and his condition at presentation was reduced with positive shock index because of exsiccosis. MCV was elevated corresponding to chronic alcoholism. Symptom of diarrhea could have been manifestation of exogenous pancreatic insufficiency (EPI), but determination of elastase as a stool marker for EPI was negative. Lipase was elevated and together with $\mathrm{CT}$ and ultrasound results alcohol induced pancreatitis was obvious.

IDH occurs mostly due to blunt abdominal trauma. ${ }^{1}$ Causes for non-traumatic hematomas such as the one described are related to the intake of anticoagulants (Warfarin, acetylsalicylic acid), coagulation disorders, malignant diseases, side effects of chemotherapy or inflammatory and autoimmune processes. ${ }^{4,10-12}$ There are rare reports of an association between IDH and acute pancreatitis. ${ }^{2,7,13}$

The pathogenesis of and relationship between IDH and pancreatitis remain unknown. Further it is unclear if pancreatitis induced intramural duodenal bleeding or duodenal hematoma is trigger for pancreatitis. ${ }^{3,8}$

Especially in cases with mild pancreatitis development of duodenal hematoma is difficult to explain. The fixed position of duodenum, its nearness to vertebral column and rich vascularization are anatomic preconditions for IDH especially in context with blunt trauma ${ }^{10}$ For non-traumatic IDH two hypotheses have been postulated. First, the presence of ectopic pancreatic tissue within the wall of the duodenum may develop acute inflammation and subsequent necrosis, and hematoma formation. Second, leakage of pancreatic enzymes in pancreatitis can injure duodenal blood vessels, namely anterior and posterior pancreatico-duodenal arcades. It may cause local necrosis and hematoma formation. ${ }^{3,4}$

The most common presenting symptoms are abdominal pain, vomiting and successive dehydration or exsiccosis. Because of the intramural bleeding the lumen of the duodenum will be obliterated and gastrointestinal passage of liquid or chime is disturbed. Gastric outlet obstruction occurs. Occasionally, patients may present with jaundice due to bile duct compression by the hematoma. On the other hand the papilla Vateri can be swollen by hematoma and the secretion of the exogenous pancreas enzymes is not possible any more resulting in pancreatitis.

Laboratory tests are of limited value for clinical diagnosis IDH. In general, a high index of clinical suspicion should be contemplated.
As diagnostic abilities, using contrastenhanced CT and magnetic resonance imaging (MRI) have improved over the last few decades. Because of its availability and cost effectiveness contrast-enhanced $\mathrm{CT}$ is recommended over MRI for detection IDH. ${ }^{3,10,14,15}$ In our case we performed native CT because of reduced GFR and elevated creatinine contrast-enhanced $\mathrm{CT}$ was impossible. But native CT scan was not able to diagnose IDH directly and could have underestimated a life threating condition retrospectively.

The changes of paradigm in management of IDH were dependent with the advent of contrast-enhanced CT. Reviewed case reports by Sorbello et al. with small bowel hematoma in context with anticoagulant therapy showed that particularly diagnostic surgery intervention declined since CT scan is available as a sufficient diagnostic tool in the mid eighties. The percentage of cases requiring surgical intervention declined from $40 \%$ in the $1981-1986$ periods, to $28 \%$ in the post 1986 period. ${ }^{16}$

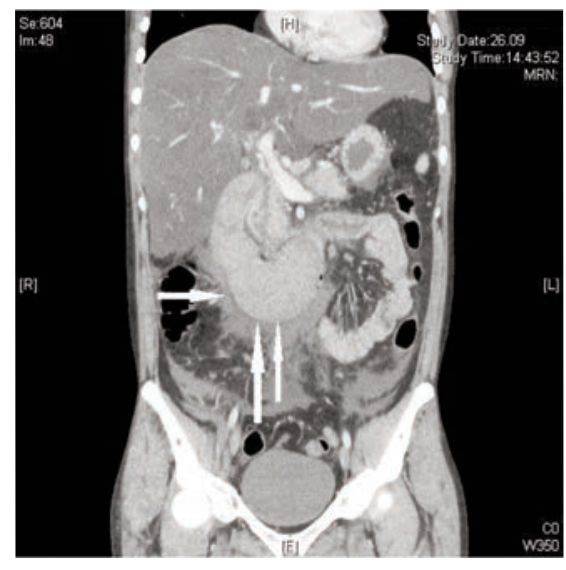

Figure 1. Extensive mass of the duodenal wall (arrows), hematoma.

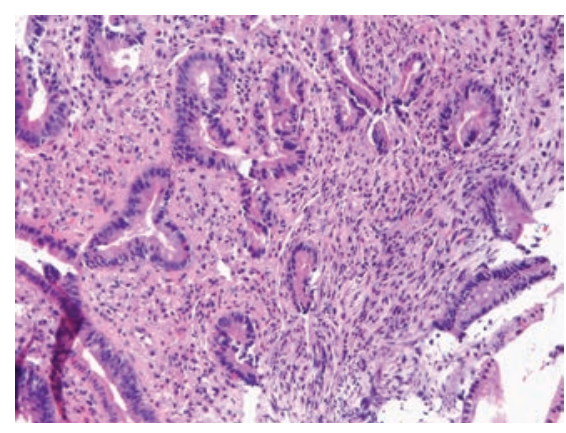

Figure 2. Endoscopic biopsy of duodenal tumor: atypical glands, adenocarcinoma not excluded (arrows). 
In events of enormous intramural bleeding perforation of duodenum occurs and life threating condition follows. In these situations surgical intervention is necessary. Furthermore surgery is indicated after 2 weeks of treatment without improvement, or in doubt of malignancy. ${ }^{4,14}$

In our case the results of native $\mathrm{CT}$ and EGD gave hints of malignant tumor confirmed by initial histological examination and finally we diagnosed IDH with EUSguided aspirate of the duodenal wall. Additional EUS is helpful and should used especially for taking biopsies in further diagnostic of duodenal or pancreatic processes. $^{2}$

In the last decade awareness of nontraumatic IDH has increased. The literature search recommends that current treatment of IDH without complications is conservative by nasogastric decompression, blood transfusion and correction of coagulation abnormalities. ${ }^{1}$

In our case IDH occurred in context with alcohol induced pancreatitis. Because of his symptoms with shock index over 1 initial intensive medical care was necessary. The gastric outlet obstruction conditioned nasogastric decompression by three luminal, jejunal tube and consisted digestive arrest. Under conservative treatment the patient recovered clinically after few days. But parts of the duodenal wall alteration were still seen in follow-up CTs 5 weeks and 6 month later while panendoscopy showed normal duodenal mucosa without luminal narrowing. Important fact was further exclusion of malignant duodenal tumor.

\section{Conclusions}

IDH is a rare pathology. Contrastenhanced CT of the abdomen and addition- al EUS are the diagnostic modalities of choice. Non-traumatic IDH can usually be managed with a conservative approach, and surgery should be reserved for complications like abdominal bleeding. Absolutely important is the exclusion of malignant origin.

\section{References}

1. Elmoghazy W, Noaman I, Mahfouz AE, et al. Surgical management of complicated intra-mural duodenal hematoma: A case-report and review of literature. Int J Surg Case Rep 2015;17:103-5.

2. Khurana T, Shah A, Ali I, et al. Intramural duodenal hematoma with acute pancreatitis in a patient with an overt pancreatic malignancy. ACG Case Rep J 2014;1:209-11.

3. Ma JK, Ng KK, Poon RT, Fan ST Pancreatic-induced intramural duodenal haematoma. Asian J Surg 2008;31:83-6.

4. Molina-Barea R, Perez-Cabrera B, Hernandez-Garcia MD, et al. [Acute abdomen due to complicated intramural duodenal haematoma. Report of a case and review of the literature]. Cir Cir 2015;83:146-50. [Article in Spanish]

5. Sirvain S, Crepeau T, Garrido JF, et al. [Anticoagulant induced intramural duodenal hematoma presenting as duodenal obstruction]. Gastroenterol Clin Biol 2008;32:611-3. [Article in French]

6. Hoenisch K, Prommegger R, Schwaighofer $\mathrm{H}$, et al. Intramural duodenal hematoma after upper gastrointestinal endoscopy. Wien Med Wochenschr 2011;161:441-4.

7. Dubois J, Guy F, Porcheron J. A pancreatic-induced intramural duodenal hematoma: a case report and literature review. Hepatogastroenterology
2003;50:1689-92.

8. Shiozawa K, Watanabe M, Igarashi Y, et al. Acute pancreatitis secondary to intramural duodenal hematoma: Case report and literature review. World $\mathrm{J}$ Radiol 2010;2:283-8.

9. Veloso N, Amaro P, Ferreira M, et al. Acute pancreatitis associated with a nontraumatic, intramural duodenal hematoma. Endoscopy 2013;45:E51-2.

10. Goyal H, Singla U, Agrawal RR. A rare cause of acute pancreatitis: intramural duodenal hematoma. Case Rep Gastrointest Med 2012;2012:275604.

11. Cheng J, Vemula N, Gendler S. Small bowel obstruction caused by intramural hemorrhage secondary to anticoagulant therapy. Acta Gastroenterol Belg 2008;71:342-4.

12. Chen PS, Cheng HC, Sheu BS. Diffuse intramural duodenal hematoma complicated by lethal necrotizing pancreatitis after endoscopic duodenal biopsy. Endoscopy 2008;40:E143.

13. Lee JY, Chung JS, Kim TH. Successful endoscopic decompression for intramural duodenal hematoma with gastric outlet obstruction complicating acute pancreatitis. Clin Endosc 2012;45:2024.

14. Chang CM, Huang HH, How CK. Acute pancreatitis with an intramural duodenal hematoma. Intern Med 2015;54:755-7.

15. Prochazka V, Marek F, Valek V, et al. Spontaneous duodenal intramural haematoma imitating pancreatic pseudocyst. Acta Chir Belg 2011;111:238-42.

16. Sorbello MP, Utiyama EM, Parreira JG, et al. Spontaneous intramural small bowel hematoma induced by anticoagulant therapy: review and case report. Clinics (Sao Paulo) 2007;62:785-90. 